\title{
Décadrages Décadrages
}

cinéma, à travers champs Cinéma, à travers champs

$12 \mid 2008$

Fredi M. Murer

\section{Compte rendu}

Zeitreisen in die Vergangenheit der Schweiz.

Auftragsfilme 1939-1959

Zurich, Praesens-Film AG, 3 DVD

\section{Pierre-Emmanuel Jaques}

\section{OpenEdition}

\section{Journals}

Édition électronique

URL : https://journals.openedition.org/decadrages/383

DOI : $10.4000 /$ decadrages.383

ISSN : 2297-5977

\section{Éditeur}

Association Décadrages

\section{Édition imprimée}

Date de publication : 10 avril 2008

Pagination : 89-98

ISBN : 978-2-9700582-7-4

ISSN : 2235-7823

Référence électronique

Pierre-Emmanuel Jaques, «Zeitreisen in die Vergangenheit der Schweiz. Auftragsfilme 1939-1959», Décadrages [En ligne], 12 | 2008, mis en ligne le 10 avril 2009, consulté le 03 avril 2022. URL : http:// journals.openedition.org/decadrages/383; DOI : https://doi.org/10.4000/decadrages.383 


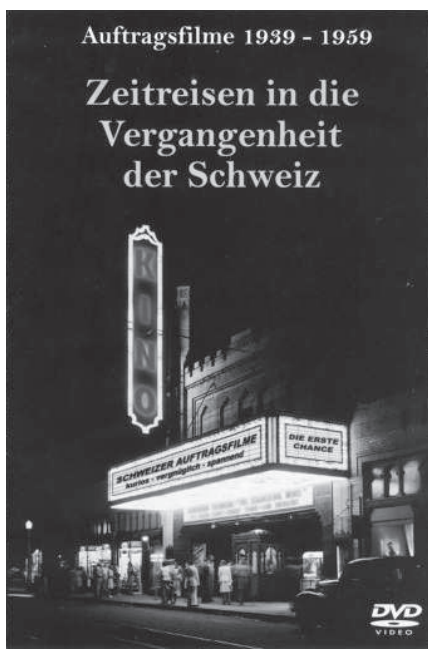

Zeitreisen in die Vergangenheit der Schweiz. Auftragsfilme 1939-1959, Zurich, Praesens-Film AG, 3 dvd

La Praesens, la plus ancienne société de production cinématographique du pays encore active, vient d'éditer une série de trois dvd consacrés à des films de commande tournés en Suisse entre 1939 et 1959. La sélection des films, les notes d'accompagnement ainsi que les recherches ayant permis leur édition ont été effectuées par Yvonne Zimmermann, maître-assistante au Seminar für Filmwissenschaft de l'Université de Zurich.

Actuellement, une séance cinématographique dans une salle commerciale consiste d'ordinaire en la projection de quelques publicités, de diverses bandes-annonces et d'un long métrage. Cette situation est en fait relativement récente, dans la mesure où une séance intégrait généralement jusque dans les années 1970 un avant-programme composé d'actualités et de courts métrages. D’autre part, certaines salles consacraient leur programmation à des films documentaires, des actualités et des dessins animés. A Genève, le Cinébref passait de tels films, alors qu’à Lausanne c'est le Cinéac qui s'était spécialisé dans ces genres. La 
fermeture de cette dernière salle en 1969 marqua la fin d'une époque pour le court métrage. Les actualités, reportages et autres documentaires passèrent dorénavant principalement à la télévision, devenue le nouveau vecteur des images éducatives et informatives. Le court métrage de fiction se trouva repoussé dans des manifestations plus ponctuelles telles que les festivals spécialisés ou les séances de type «ciné-club». Le phénomène de la commande ne disparut pas pour autant: la télévision continua à demander à des cinéastes de fournir des reportages, notamment des émissions comme Continents sans visa, l'ancêtre de Temps présent. Les liens de la télévision par rapport à la production cinématographique ont aussi largement évolué par la suite, dans la mesure où la télévision a été amenée à jouer un rôle toujours plus important, rôle renforcé par l'adoption du "pacte de l'audiovisuel»: la SSR-Idée suisse figure ainsi au générique de nombreux films et est devenue un partenaire majeur de la production audiovisuelle entendue en un sens large. Par ailleurs, les entreprises et les organismes touristiques firent aussi appel à des cinéastes pour réaliser des films présentant leurs activités ou vantant telle ou telle région. Ils continuent à demander à des cinéastes de leur fournir des images, de manière à alimenter, entre autres, leurs sites internet. Ainsi, pour un pays dont la production cinématographique était et reste relativement limitée, les films de commande revêtent une grande importance. Garantissant aux sociétés de production des commandes régulières, ceux-ci permettaient aux techniciens (opérateurs, monteurs, etc.) et aux laboratoires de survivre malgré le nombre peu élevé de longs métrages de fiction réalisés chaque année. Si la plupart des entreprises cinématographiques produisaient aussi bien des fictions que des documentaires, certaines se spécialisèrent dans ce dernier domaine, comme CentralFilm, créé dans les années 1930. Cette filiale de la Praesens se rendit rapidement indépendante en se consacrant exclusivement aux documentaires ou aux publicités. Elle poursuivit dans ce domaine pour ne disparaître que récemment, étant intégrée dans la nouvelle société Cinecom, le principal fournisseur de publicités pour les cinémas de Suisse.

Les trois dvd comportent pour l'essentiel des films de la Praesens (ou de Central-Film) et de Gloriafilm. Ces sociétés comptaient parmi les plus importantes maisons dans le domaine, avec Condor-Film et Pro Film. D'autres firmes telles que Kern-Film ou Charles Zbinden - dont un film figure cependant sur l'un des dvd - ont aussi réalisé de nombreux films de commande. Cette limitation, liée à des questions de droits d'auteurs - la Praesens a racheté les droits de Gloriafilm - ne provoque cependant pas une impression de restriction dans la mesure où les films rassemblés font preuve d'une grande diversité. 
Cette diversité se manifeste d'abord sur un plan temporel : le film le plus ancien (L'beure /Die Stunde) remonte à 1939, alors que le plus récent date de 1958. Les 27 films s'échelonnent d'ailleurs sur l'ensemble de cette période, avec une proportion légèrement plus forte pour l'immédiat après-guerre (douze films entre 1946 et 1950). Il est difficile de déterminer si cette concentration repose sur la disponibilité des copies ou sur le fait que cette période constituait un contexte de production particulièrement important en termes quantitatifs. Selon les chiffres fournis par l'Office fédéral de la statistique ${ }^{\mathbf{1}}$, la période comprise entre 1938 et 1942 voit paraître un nombre plus important de courts métrages (plus de 100) pour baisser légèrement puis reprendre et s'accroître progressivement. Si les sources qui sont à l'origine de ce décompte demeurent mystérieuses, celui-ci indique néanmoins des tendances intéressantes. L'augmentation du nombre de films correspond à la période précédant immédiatement la Seconde guerre; cette augmentation fléchit légèrement durant la guerre avant de s'accroître régulièrement. Il conviendrait d'analyser plus précisément l'influence exercée par la mise en place d'une politique de défense nationale spirituelle et d'examiner les causes qui ont pu mener à une augmentation de la production après-guerre, en prenant en compte le public visé, entre autres. Il faudrait aussi déterminer la corrélation qui peut être établie entre la production de fictions et de documentaires. A première vue, il semble difficile d'établir un lien direct au vu du nombre limité de longs métrages de fiction produits durant ces années.

De la même manière que pour la fiction, se pose également la question du public visé. Alors que nombre de films visaient en premier lieu un public «local», certains étaient destinés à toucher un public plus large, international. Plusieurs courts métrages figurant sur les trois dvd s'adressent vraisemblablement à un public qui n'est pas strictement local, tels que Reise nach dem Süden (Georges C. Stilly, 1958, Gloriafilm) qui vante le projet de tunnel du Grand-Saint-Bernard qui facilitera le déplacement du Nord au Sud. D’autres titres visaient manifestement un public plus déterminé, sans pour autant être centrés sur des éléments trop particuliers: des films professionnels comme ceux de la Praesens ou de Gloriafilm devaient pouvoir circuler largement au vu des coûts engagés. Les films destinés à une circulation restreinte étaient confiés plutôt à des sociétés de moindre envergure et étaient généralement tournés en $16 \mathrm{~mm}$, parfois même en muet.

Les films rassemblés sur ces trois dvd peuvent être classés selon trois catégories principales: le film touristique, le film industriel et le film sociétal. Si les deux premières catégories correspondent à des ensembles
1 Communiqué de presse du 17.04.2008, $n^{\circ}$ 0351-0803-40. 
Das Gesetz der Strasse (1946)

2 "L'OSEC et la propagande par le film", dans Informations économiques, $\mathrm{n}^{\circ} 31,2.08 .1939$, [p. 153].

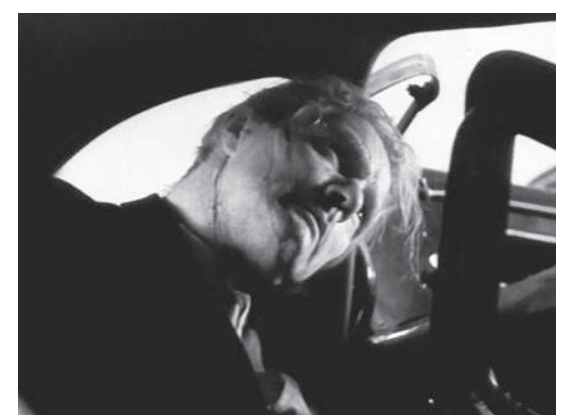

clairement définis, dont l'origine remonte aux débuts du cinéma, la dernière, que je forge artificiellement, renvoie à des titres liés aux préoccupations d'une période ou à un aspect de la vie sociale. On trouve ainsi un film consacré aux accidents de la route et à leur prévention, Das Gesetz der Strasse (Max Haufler, 1946, Praesens), prévention qui peut aussi être appliquée à Eine Freudin in der grossen Welt (Kurt Früh, 1958, Gloriafilm), mais concernant un danger d'une autre nature : en l'occurrence celui qui menace les femmes et que les Amies de la jeune fille s'efforcent d'endiguer. Au sein même de ces trois ensembles, on trouve des films marqués par de grandes différences. A lui seul, le domaine industriel présente ainsi une grande diversité. Die Stunde/L’heure (1939) est un film réalisé par la Praesens à la demande de l'Office suisse d'expansion commerciale (OSEC) en collaboration avec la Chambre suisse de l'horlogerie 2 . Il est réalisé par Robert Chessex, un employé de l'OSEC, responsable du domaine cinématographique, qui signe le scénario. Les prises de vues furent confiées à Emil Berna, l'opérateur en charge de la plupart des longs métrages de fiction de la Praesens. L’heure est réalisé pour passer en premier lieu à l'Exposition nationale de Zurich en 1939. En plus des traditionnelles versions allemande et française, le film a été doublé en anglais, italien, portugais et espagnol. Il est ainsi projeté dans le Pavillon suisse de l'Exposition mondiale de New York avant de circuler en avantprogramme dans les salles du pays et à l'étranger. Il figure encore dans une exposition suisse (à Buenos Aires) et dans le cadre d'une foire (à Lyon). Il est présenté aux Etats-Unis grâce au relais fourni par des associations professionnelles liées à l'horlogerie. Une centaine de projections sont organisées en Algérie. Les consulats, légations, chambres de commerce et autres agences commerciales le feront circuler en Amérique latine et au Moyen Orient (Iran et Egypte). Enfin, en Suisse, des projections scolaires sont organisées par la Semaine suisse, un organisme constitué en vue de promouvoir l'économie nationale. Il est évident 
que cette circulation intensive a assuré à ce film une diffusion bien plus importante qu'à la plupart des longs métrages de fiction produits à la même période dans le pays. La vision de ces films démontre aussi leur importance sur le plan de la représentation nationale. Ces courts métrages ont certainement participé à l'alimentation de certains clichés nationaux, notamment ceux liés au travail ou au système politique.

Le secteur touristique a aussi largement contribué à répandre une image idyllique de la Suisse, comme en atteste Euses Bähnli (Josef Dahinden, 1942, Praesens). Le film est réalisé à la demande d'Hotelplan, l'agence de voyages de la Migros. Gottlib Duttweiler avait acquis en 1941 ce train qui gravit le Monte-Generoso alors que son exploitation était en arrêt depuis 1939. Le funiculaire est ainsi rendu au peuple suisse, qui peut parcourir et découvrir son pays - pour reprendre le slogan touristique contemporain: "Va et découvre ton pays». L'unité nationale sera ainsi raffermie et l'amour de la patrie renforcé grâce à l'émotion suscitée par la vision des paysages locaux.

Réalisé quelques années plus tard, Luzern und seine Internationalen Musikfestwochen (1946, Gloriafilm) de Hans Trommer vise plutôt un public international. On cherche manifestement à redonner un statut de centre touristique international à Lucerne et au lac des Quatre-cantons. Jouant avec les clichés - tous les monuments de la région sont représentés -, le film cherche aussi à renouveler le genre en faisant jouer musique et images, notamment lors de séquences consacrées à l'interprétation de certaines compositions. Le montage suit le rythme musical d'une pièce jouée à l'orgue. Plus tôt dans le film, les paysages bordant la maison où Wagner a séjourné au bord du lac des Quatre-cantons servent de contrepoint visuel à la musique. En comparaison, Ski-Frübling in Grindelwald (1948, Gloriafilm), réalisé par Adolf Forter, apparaît plus proche de l'esthétique des affiches et des brochures que les promoteurs touristiques ont développée parallèlement ${ }^{3}$. Le pittoresque prédomine dans

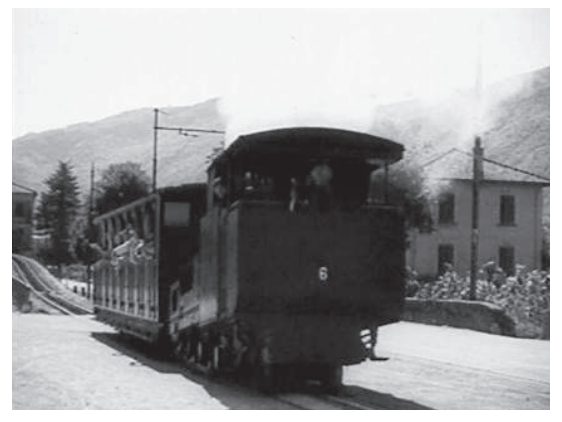

Euses Bähnli (1942)
3 Jean-Charles Giroud, Michel Schlup (éd.), Paradis à vendre. Un siècle d'affiches touristiques suisses, Patrick Cramer Editeur / Associations des Amis de l'affiche suisse, Neuchâtel/Genève, 2005. 


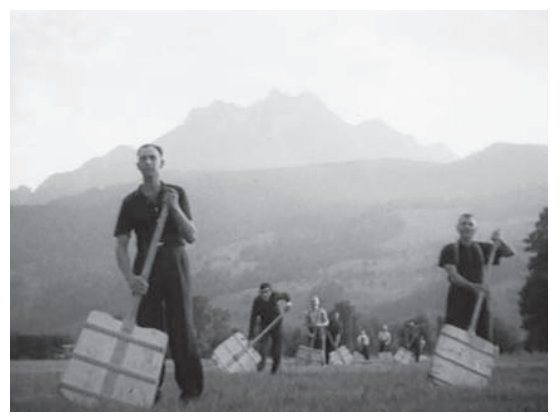

Luzern und seine internationalen Musikfestwochen (1946)
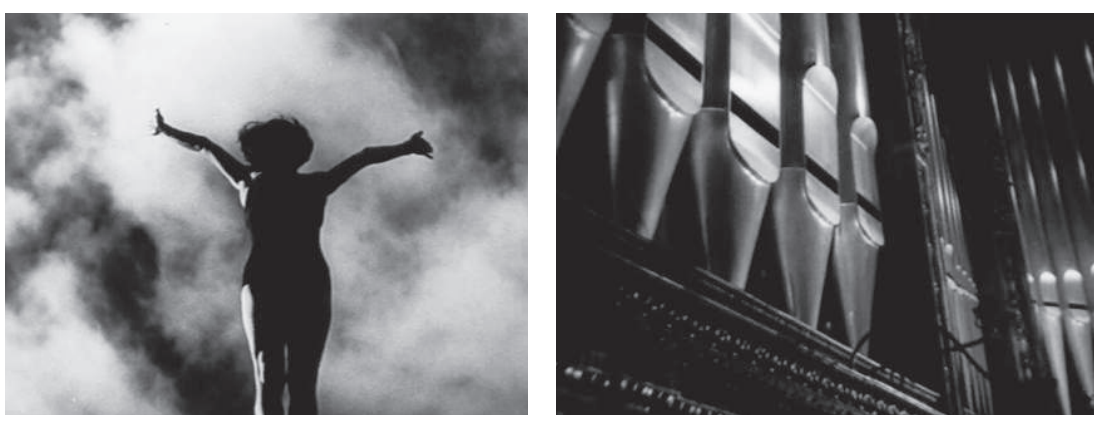

des images qui ont pour charge d'évoquer le charme des sommets enneigés, où déjà pointent les premières fleurs. Le film est placé sous le signe de la nature enchanteresse pour promouvoir Grindelwald et le train menant aux pistes (Firstbahn).

Outre l'Office national suisse du tourisme et les différentes stations, les CFF et les PTT furent parmi les plus importants commanditaires de films touristiques. Les CFF furent ainsi à l'origine de Das verwechselte Bild (Alfred Bruggmann, 1958, Praesens). Un des responsables de la publicité des CFF, Werner Belmont, cosigne le scénario de ce film qui comporte une courte narration. Un détective privé doit retrouver une starlette se reposant en Suisse. En plus des paysages, le confort des trains et la qualité des services proposés dans les wagons-restaurants et les buffets de gare sont évoqués au passage. La présence d'acteurs bien connus comme Walter Roderer, déjà célèbre au cabaret et au théâtre, sert de base à un film destiné à véhiculer un message promotionnel. Schaggi Streuli apparaît pour sa part dans Demokratie in Gefahr (Kurt Früh, 1949, Praesens) où il incarne un citoyen victime d'un état totalitaire. Dans Der "Geist» von Allenwil (Max Haufler, 1950, Praesens), Streuli réinterprète son personnage de policier, qui lui avait valu un immense succès radiophonique. La courte enquête qu'il mène permet d'introduire un commentaire sur la lessive Radion.

L'apparition de ces éléments de fictionnalisation est une des caractéristiques de la période. Si l'on trouve déjà des scènes reconstituées dans les films qualifiés de documentaires dans les années 1920 et 1930, leur proportion augmente très nettement après-guerre. Dans Das Gesetz der Strasse, à la suite d'un accident routier, nous assistons à une scène de reconstitution qui emprunte la forme d'une courte narration: le conducteur fautif a bu quelques verres dans une auberge de campagne et roule à tombeau ouvert. Sa course folle conduit à une collision avec 
un taxi. Une ambulance emporte les victimes. Cette courte narration sert d'exemple à un discours plus large qui porte sur la prévention routière. On assiste à un même phénomène d'exemplification dans Das Tal der reissenden Wasser (Aldolf Forter, 1953, Gloriafilm), où la construction d'un barrage apporte la prospérité à une vallée reculée. La fictionnalisation apparaît aussi souvent dans le cas de construction de véritables personnages comme celui du détective dans Das verwechselte Bild. Le phénomène se retrouve dans Unser Mitbürger Christian Caduff (Kurt Früh, 1955, Gloriafilm) où, pour présenter une entreprise chimique, on a choisi de se concentrer sur un ouvrier clairement personnalisé. Son parcours apparaît comme exemplaire dans la mesure où il correspond à celui de nombreux autres employés : en tant que paysans, ils n'arrivaient plus à obtenir un revenu suffisant. Le travail en usine leur a permis de rester dans leur région. L'industrie hors des centres urbains apparaît ainsi comme une possibilité de lutter contre l'exode rural.

Les 27 courts métrages rassemblés ici construisent une représentation relativement homogène du pays. Même quand certains titres sont liés à un moment particulier (la Landi), ils s'efforcent de développer un discours porteur de valeurs plus générales. Die Äcker der Industrie (Hermann Haller, 1945, Gloriafilm) porte sur l'implication des industries dans le plan d'extension des cultures (Anbauschlacht), connu sous le nom de plan Wahlen. Mais plus généralement, le film suggère qu'ouvriers et patrons se trouvent unis dans un intérêt général qui dépasse toute opposition de classe. Mensch und Maschine (Adolf Forter, 1955, Gloriafilm) participe d'un même discours en insistant sur le lien à la terre et au lieu d'origine: les ouvriers ont pu rester dans leur ferme tout en trouvant un emploi dans la métallurgie. Un commentaire suggère que l'harmonie régnante est née de l'esprit de liberté qui constitue le fondement de l'Etat helvétique. Le fonctionnement de la démocratie directe est clairement explicité dans Der Souverän (Franz Schnyder, 1947, Praesens), tourné dans l'Emmental: on assiste à une assemblée du conseil communal où l'on discute des mérites et des défauts de l'instituteur. Ces mêmes firmes ont tourné des films à la demande des syndicats: Lasst uns tapfer beginnen (Adolf Forter, 1947, Gloriafilm) constitue un plaidoyer en faveur de l'Assurance vieillesse (AVS), alors que Für eine bessere Zukunft (1953, Gloriafilm) promeut l'engagement syndical en expliquant les avantages offerts par l'organisation.

Les films rassemblés sur ces trois dvd portent pour la plupart la signature d'une personnalité ayant aussi œuvré dans le domaine de la fiction. Sigfrit Steiner signe ainsi quelques longs métrages, dont MaturaReise (1942) et Steibruch (1942), de même que Max Haufler qui réalise L'or 
Mitenand gahts besser! (1949)

dans la montagne (1938) d'après Farinet ou la fausse monnaie de Ramuz, puis Menschen, die vorüberziehen... (1942). Parmi les réalisateurs de ces films de commande, on trouve aussi Hans Trommer et Valerian Schmidely, célèbres maintenant pour leur Romeo und Julia auf dem Dorfe (1941), considéré comme une des principales réussites du cinéma suisse. Les échanges entre fiction et documentaire sont ainsi monnaie courante et garantissent une continuité et un avenir dans la carrière des réalisateurs, mais aussi du personnel technique. Les opérateurs, les musiciens, les scénaristes, les monteurs sont les mêmes dans les deux domaines: Emil Berna figure aux génériques de nombreux films, avant tout ceux de la Praesens, de même que Robert Blum qui écrit de très nombreuses partitions. René Martinet effectue le montage à la Gloriafilm sans distinction entre documentaire et fiction, courts ou longs métrages. Il n'existe donc pas de barrière entre le domaine de la fiction et celui de la commande.

Par rapport aux pays voisins, dotés d'une industrie cinématographique, le passage d'une forme à l'autre constitue un phénomène courant. Pour prendre un exemple, le documentaire assure manifestement la formation de Kurt Früh : après avoir débuté à la Central-Film, il réalise une série de courts métrages pour la Praesens, dont Demokratie in Gefahr (1949), une mise en garde contre le totalitarisme à l'esthétique travaillée (angles obliques, déformations). Après avoir réalisé Polizischt Wäckerli (1954), son premier long métrage de fiction, il n'abandonne pas pour autant le film de commande: il signe ainsi Eine Freudin in der grossen Welt (1955) ou encore Unser Mitbürger Christian Caduff (1955). Par contre, d'autres personnalités n'ont manifestement pas cherché à approcher le domaine de la fiction, comme Adolf Forter, qui après avoir été en charge de l'Armee Film Dienst devient l'un des principaux réalisateurs de films de commande auprès de Gloriafilm. Son rôle semble d'ailleurs avoir été plus celui d'un directeur de la production documentaire que celui d'un réalisateur. Il œuvre dans tous les domaines, du film touristique

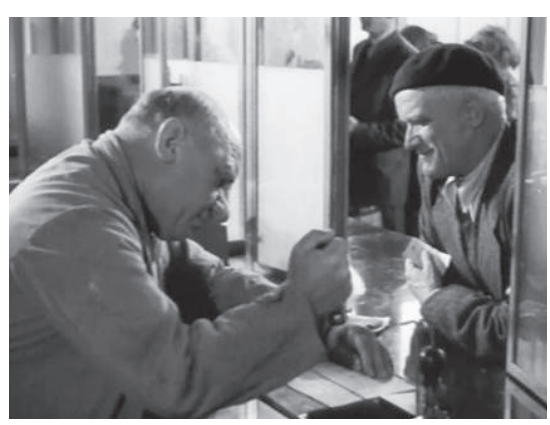


(Ski-Frübling in Grindelwald, 1948) au film industriel (Der Schweizer Pneu, 1949), en passant par le "Propagandafilm» en faveur de Caritas: Das Grösste aber ist die Liebe (1950). Ses films comportent parfois de petites narrations comme Mitenand gahts besser! (1949), destiné à appuyer l'adoption d'une loi sur le statut des fonctionnaires fédéraux. De même, Josef Dahinden demeure un adepte du documentaire, de ses premiers films de skis à la fin des années 1920 jusqu'à ceux qu'il réalise dans les années 1950, tout en cherchant à renouveler le genre.

Si l'existence des ces films de commande est connue des spécialistes, les occasions de les découvrir demeurent néanmoins rares. Jusqu'à récemment, seuls quelques titres ont circulé à diverses occasions, comme Pulver (1944) de Herbert E. Meyer, réalisé à l'initiative du Service des films de l'armée et qui explicite la fabrication de la poudre. Une copie avait été restaurée par la Cinémathèque suisse en 1977 et avait quelque peu circulé. Le montage alerte du film, l'insistance sur le danger, sa construction efficace lui assurent un intérêt dont n'a joui qu'une infime proportion de films de commande. Généralement, ce genre était considéré comme peu attrayant et les films jugés intéressants l'étaient dans la mesure où leurs "auteurs" s'efforçaient d'échapper à la commande qui leur était imposée. Ce sont exactement de tels jugements qui furent prononcés à l'encontre des court métrages de Max Haufler. Dans le volume paru en 1982, Max Haufler. Der Maler, Schauspieler, Filmautor und

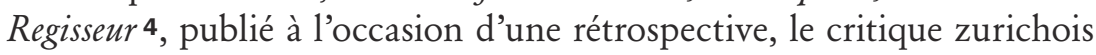
Martin Schlappner passe en revue les films de commande réalisés par Haufler qu'il semble peu apprécier, à l'exception de Das Gesetz der Strasse (1946) et Venezianische Rhapsodie (1948) parce qu'ils lui paraissent justement subvertir la commande. Ce jugement est cependant difficile à soutenir dans la mesure où l'inventivité et le soin constant dans la réalisation de Das Gesetz der Strasse (1946), loin de subvertir la commande, contribuent bien plutôt à l'efficacité du message véhiculé. Trop souvent, on a eu tendance à estimer que si tel ou tel film de commande était bon, c'était parce qu'il transgressait la commande. Or, comme le montre les renseignements collectés par Yvonne Zimmermann dans les compléments des $\mathrm{dvd}$, ces films sont le résultat de négociations précises. Les commanditaires sont généralement satisfaits des films fournis par les sociétés contactées. Ce qui est corroboré par l'usage récurrent des films, généralement sur une longue période. Le fait qu’une personnalité comme Max Haufler se voit confier la réalisation de plusieurs films de commandes témoigne de la satisfaction que son travail suscitait auprès de son employeur (Gloriafilm). On a eu trop souvent une vision
4 Bea Cuttat, Mathias Knauer (éd.), Max Haufler. Der Maler, Schauspieler, Filmautor und Regisseur, Schweizerisches Filmzentrum, Zurich, 1982 (Texte zum Schweizer Film 6). Le passage où Martin Schlappner mentionne les films de commande de Haufler se trouve pp. 113-114. 
5 Gérard Leblanc, "L'auteur face à la contrainte", dans Jean-Pierre Bertin-Maghit, Béatrice Fleury-Vilatte (éd.), Les institutions de l'image, Ecole des Hautes Etudes en Sciences Sociales, Paris, 2001, pp. 75-84.

6 Schaufenster der Nation, Der nicht-fiktionale Film in der Schweiz 1896-1964 - Vitrine de la nation: le film documentaire en Suisse 18961964, à paraître chez Limmat Verlag (Zurich). Le livre comportera des textes signés Yvonne Zimmermann, Pierre-Emmanuel Jaques, Anita Gertiser. II est le résultat d'une recherche menée sous l'égide du Fonds national de la recherche scientifique, dirigée par Margrit Tröhler (Seminar für Filmwissenschaft). caricaturale du rôle des commanditaires, envisagés comme des entités dénuées de bon sens et forcément réfractaires à toute innovation. Au vu des films rassemblés ici, il convient de sortir de cette vision pour adopter un point de vue plus complexe, comme Gérard Leblanc nous invite à le faire en parlant de cahier des charges pour désigner le rapport qui s'établit entre commanditaires et cinéastes $\mathbf{5}$. La contrainte est ainsi relative et doit être examinée au cas par cas. C'est d'ailleurs là le propos des bonus annexés aux films, composés de textes qui rendent compte de la commande à l'origine des films, de leur circulation ou qui témoignent de leur accueil critique. Parfois figurent aussi des documents photographiques, rares témoignages de la légèreté des moyens à disposition (absence de prise de son directe, équipe technique réduite), mais aussi du souci de fournir un point de vue documenté (recours au travelling, installation de la caméra à des emplacements surélevés).

Ces trois dvd ouvrent une nouvelle perspective sur le cinéma suisse, considéré le plus souvent en fonction de ses seuls longs métrages de fiction: ils font ainsi apparaître un pan central de son économie et de ses réalisations. Ne se contentant pas de mettre en évidence cet aspect méconnu, l'étude de ces films revêt un intérêt majeur dans la mesure où nombre de ceux-ci ont façonné la représentation nationale. Commandités par des organismes subventionnés par l'Etat, ils ont été largement mis au service de la diffusion d'une image du pays. Au-delà ou en deçà de l'inscription omniprésente de paysages de montagne, ils ont participé à la représentation identitaire de la Suisse à laquelle les CFF et les PTT ont contribué de manière évidente. Mais aussi plus largement, ils attestent de valeurs que l'on cherche à faire passer comme naturelles et généralement partagées par le pays, telles que le goût du labeur, le besoin d'innovation, une certaine idée humanitaire et une stabilité politique loin des oppositions divisant les autres pays. La publication prochaine d'un ouvrage consacré à ces aspects devrait apporter un éclairage supplémentaire sur ce corpus encore peu étudié 6 . 